\title{
Full Field X-Ray Imaging - Microanalysis between Table Top and Free Electron Laser Experiments
}

\author{
H.Soltau, * R.Hartmann, * A.Hartmann,* P.Holl,* S.Ihle, ${ }^{*}$ Ch.Thamm,* A.Liebel, ${ }^{* *}$ I.Ordavo, ** \\ G.Schaller, ${ }^{++}$F.Schopper, ${ }^{++}$L.Strüder, ${ }^{++}$ \\ *PNSensor GmbH, Römerstr. 28, D-80803 München, Germany \\ **PNDetector GmbH, Emil-Nolde-Str.10, D-81735 München, Germany \\ ${ }^{++}$MPI Halbleiterlabor, Otto-Hahn-Ring 6, D-81739 München, Germany
}

X-Ray imaging experiments move into the focus of biology, chemistry and material analysis. For this purpose new detectors and methods are developed worldwide. The common goal of the investigations is an $\mathrm{x}$-ray image acquired significantly fast and simple, with adequate spatial resolution, delivering information on the material structure or the elemental composition of the object preferably in its original shape.

While usually such an image is achieved by sequential probe mapping by using a scanning microbeam of electrons or x-rays, collecting the fluorescence photons by a one-dimensional energy dispersive detector here the alternative of irradiating the probe with a broad x-ray beam and recording simultaneously the fluorescence photons with a two-dimensional energy dispersive detector is presented. Such we are talking on full-field XRF imaging.

Recently at the hard X-ray free-electron laser (FEL) at the Linac Coherent Light Source (LCLS) diffraction single shots have been collected from a hydrated stream of biological macromolecules using femtosecond pulses. The problem of radiation damage which is often limiting the resolution in biological imaging has been mitigated by using x-ray pulses shorter than the time-scale of the damage process itsself. The feasibility of the concept of "diffraction before destruction" has been proven by the experiments at the new FELs with resolution lenghts within the sub-nanometre range. High quality $3 \mathrm{D}$ structure factor data have been revealed for Photosystem I, a very large membrane protein complex [1].

The detector which has been used for that purpose is a new kind of CCD named pnCCD which has been originally developed for extraterrestrial physics and which is now intoduced into the world of material analysis. The pnCCD is avoiding active MOS structures for reasons of radiation hardness. Besides the outstanding property of radiation hardness the detector is characterized by its high quantum efficiency - as the well-known Silicon Drift Detector (SDD) the pnCCD is a truly fully depleted detector with a sensitive thickness of $450 \mathrm{um}$ and a homogenous radiation entrance window adapted to the detection of low x-ray energies down to $100 \mathrm{eV}$. The pixel sizes of the pnCCDs vary between $36 \mathrm{um}^{2}$ and $150 \mathrm{um}^{2}$ providing spatial resolutions down to $2 \mathrm{um}$ in the single photon mode using the center of mass calculation method. All CCD columns are operated in parallel read out mode providing high imaging rates up to 1000 images per second. The noise level of the imager is very low with 2.5 ENC delivering excellent spectroscopic data.

Figure 2 shows the very large pnCCDs with a pixel size of $75 \mu \mathrm{m}$ and an overall area of $60 \mathrm{~cm}^{2}$ which are used for the diffraction experiments at LCLS. The detectors have a hole in the middle for the exciting X-ray beam. They had been integrated as a relevant part into the multi-purpose CAMP Camera [2]. In contrast in Fig. 1 a plain and compact X-ray Color Camera is presented which allows 
$\mathrm{x}$-ray imaging at table top level by help of a x-ray microfocus tube and a polycapillary optic in front of the detector. It is equipped with a split frame $48 \mathrm{um}$ pnCCD with an active area of $2 \mathrm{~cm}^{2}$.

In the talk the relevant design and performance features of the x-ray imagers will be outlined as well as measurement results be presented ranging from table top experiments to the most interesting results of CAMP at the FEL within the last two years [1], [3].

[1] H. N. Chapman et al, Nature 470 (2011) 73.

[2] L. Strüder et al, NIMA 614 (2010) 483.

[3] M. M. Seibert et al, Nature (2011) to be published.
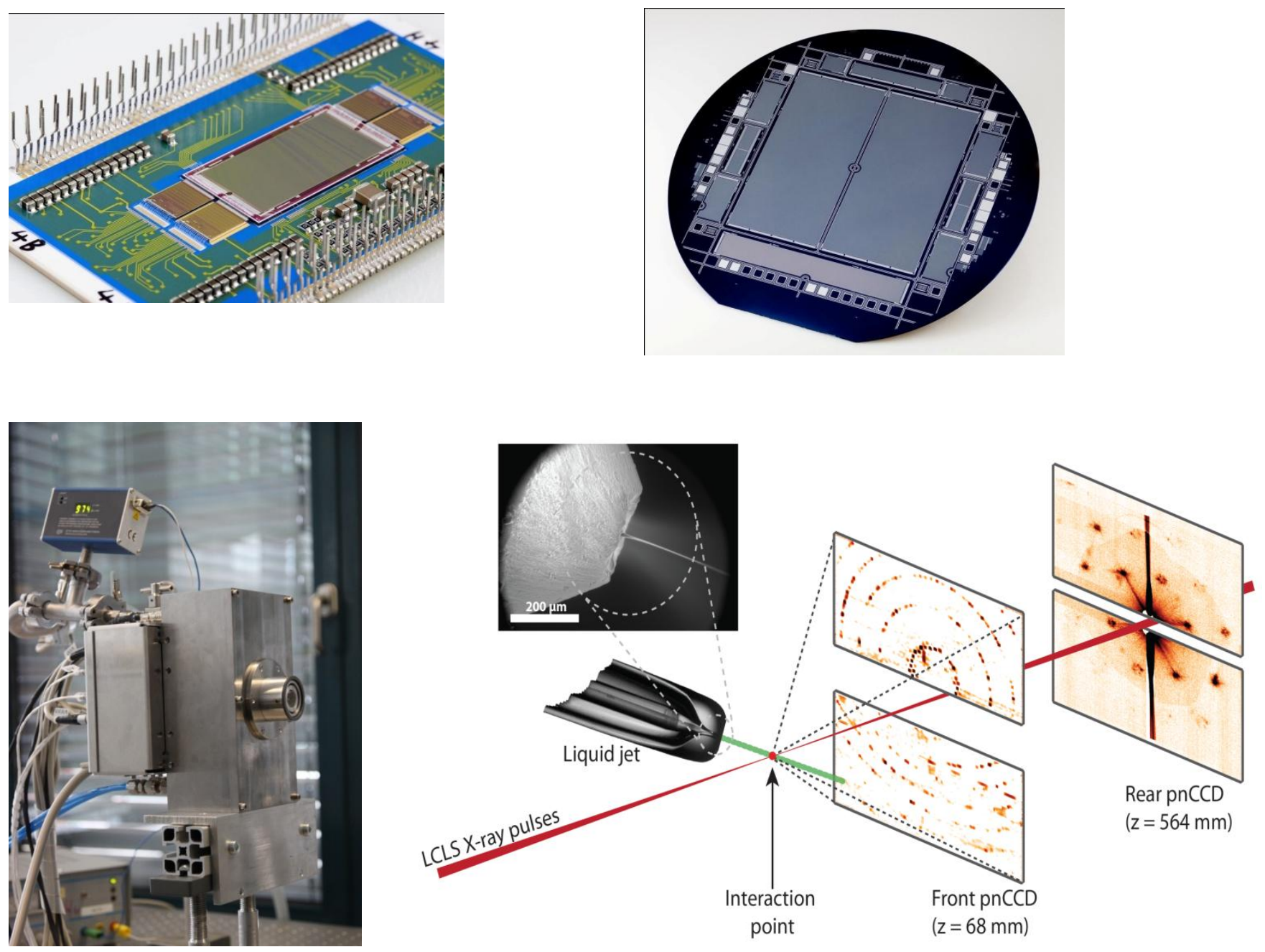

FIG.1. Compact X-ray Camera. A high speed split frame pnCCD with a pixel size of $48 \mathrm{um}$ and an image rate of 400 to 1.000 frames per second is integrated into a compact portable X-ray camera.
FIG 2: Femtosecond Nanocrystallography with the CAMP Camera. In this experiment two pairs of very large pnCCDs with an area of $60 \mathrm{~cm}^{2}$ each record low- and high-angle diffraction patterns from single X-ray FEL pulses hitting randomly nanocrystals injected perpendicular to the beam. 